\title{
Risk Factors for Bone Loss with Prostate Cancer in Korean Men Not Receiving Androgen Deprivation Therapy
}

\author{
Sun-Ouck Kim, Taek Won Kang, Dongdeuk Kwon, Kwangsung Park, Soo Bang Ryu \\ Department of Urology, Chonnam National University Medical School, Gwangju, Korea
}

\begin{abstract}
Purpose: Preexisting bone loss in men with prostate cancer is an important issue due to the accelerated bone loss during androgen deprivation therapy (ADT). In addition, a high prostate-specific antigen (PSA) level has been reported to be related to bone metabolism. This study assessed the factors associated with osteoporosis in Korean men with non-metastatic prostate cancer before undergoing ADT.

Materials and Methods: The study enrolled patients admitted for a prostate biopsy because of a high PSA or palpable nodule on a digital rectal examination. We divided the patients $(\mathrm{n}=172)$ according to the results of the biopsy: group I, non-metastatic prostate cancer $(n=42)$ and group II, benign prostatic hypertrophy $(\mathrm{BPH} ; \mathrm{n}=130)$. The lumbar bone mineral density (BMD) was evaluated using quantitative computed tomography. The demographic, health status, lifestyle, body mass index (BMI), serum testosterone concentration, and disease variables in prostate cancer (Gleason score, clinical stage, and PSA) were analyzed prospectively to determine their effect on the BMD.

Results: The estimated mean T-score was higher in group I than in group II (-1.96 \pm 3.35 vs. $-2.66 \pm 3.20)$, but without statistic significance $(p=0.235)$. The significant factors correlated with BMD in group I were a high serum PSA $(\beta=-$ $0.346, \mathrm{p}=0.010)$ and low BMI $(\beta=0.345, \mathrm{p}=0.014)$ in the multiple linear regression model. Also old age $(\mathrm{r}=-0.481, \mathrm{p}=$ $0.001)$, a high serum PSA $(r=-0.571, p<0.001)$, low BMI $(r=0.598, p<0.001)$, and a high Gleason's score $(r=-0.319$, $\mathrm{p}=0.040)$ were the factors related to BMD in the correlation. The significant factors correlated with BMD in group II were old age $(\beta=-0.324, p=0.001)$ and BMI $(\beta=0.143, p=0.014)$ in the multiple linear regression model.

Conclusions: The risk factors for osteoporosis in men with prostate cancer include a low BMI, and elevated serum PSA. Monitoring BMD from the outset of ADT is a logical first step in the clinical strategy to avoid or minimize potential bonerelated complications in these patients.
\end{abstract}

Key words: prostate neoplasm; osteoporosis; androgen deprivation therapy; prostate specific antigen Int Braz J Urol. 2009; 35: 183-9

\section{INTRODUCTION}

In men, $36 \%$ of osteoporosis is due to low androgen levels, which can occur with congenital hypogonadism, the aging process, or androgen deprivation therapy (ADT) for the treatment of advanced prostate cancer (1). Bone is the most common site of metastasis in many types of cancer, including advanced prostate cancer. Several studies have reported that ADT can induce bone loss and increase bone fracture risk in men with prostate cancer (2-4). Moreover, many other authors have reported that patients with prostate cancer had previously developed osteoporosis before ADT compared to non-prostate cancer patients (5). This suggests that factors beside ADT may cause osteoporosis in prostate cancer, but 
no data on this subject has been reported for Korean patients with prostate cancer.

Recently, the increased life expectancy, advanced diagnostic techniques, and Westernized eating habits have contributed to a high incidence of prostate cancer in Koreans and an increased mortality rate due to co-morbidity. Thus, predicting and preventing the progression of osteoporosis in patients with prostate cancer is of critical importance. Before initiating ADT, it is necessary to identify the causes of bone loss and related risk factors for osteoporosis. However, who should undergo bone mineral density (BMD) testing before ADT remains unclear. There is a major need to determine ways to treat patients with prostate cancer undergoing ADT without increasing the risk of osteoporosis. This study evaluated the factors associated with osteoporosis in patients with nonmetastatic prostate cancer before undergoing ADT as compared to those with benign prostate hypertrophy (BPH) alone.

\section{MATERIALS AND METHODS}

After informed consent was obtained from all patients, a prospective trial was initiated at Chonnam National University Hospital from January to December 2005. This study enrolled patients hospitalized for a prostate biopsy because of a high PSA or palpable nodule on rectal examination. Based on previous medical history and physical examination, patients with thyroid or parathyroid disease, uncontrolled diabetes mellitus, cardiovascular disease, digestive disorders, and chronic steroid users were excluded, as well as patients found to have bone metastasis on plain film $\mathrm{X}$-rays and a bone scan. Patient information on demographics, health status, lifestyle, tobacco use, and body mass index (BMI) were obtained. The patients were divided into two groups according to the result of the prostate biopsy: group I, patients with prostate cancer $(n=42)$, and group II, patients with BPH $(n=$ 130). The general conditions of the patients assessed according to performance status were good and they reported light physical activity and moderate intakes of calcium, alcohol, and caffeine. We evaluated the relationship between the patient characteristics and disease variables. This was analyzed prospectively us- ing univariate and multivariate methods to determine their role in the BMD levels previously established using quantitative computed tomography (QCT) of the lumbar spine. The Institutional Review Board at our hospital approved the study.

\section{Prostate Cancer Disease Variables}

The patients' charts were reviewed to obtain information on clinical variables pertaining to prostate cancer: clinical stage, Gleason score, and PSA. To measure PSA (Access Assay, Hybritech) and total testosterone (Immunoenzymatic assay, Beckman), serum was obtained at between 08:00 and 09:00 h.

\section{Bone Mineral Density}

The BMD in L1-4 was measured using QCT. Using the World Health Organization Criteria, a normal BMD was defined as one greater than -1 standard deviation (SD) below the young adult mean value (T-score), osteopenia as a T-score between -1 and -2.5 SDs, and osteoporosis as a T-score of -2.5 or less (6).

\section{Statistical Analysis}

Descriptive, comparative, univariate, and multivariate analyses using the Statistical software package for the Social Sciences, version 12.0 (SPSS Inc., Chicago, IL) were performed to describe BMD and the associations between it and the disease variables. Simple correlation analysis was performed using the nonparametric Spearman correlation coefficient. An independent samples t-test was used for comparison analysis. Variables statistically significant in the univariate analysis were included in the multiple linear regression model with BMD of the lumbar spine as the dependent variable. Two-tailed tests were used for all correlation and comparison analyses. $P$ values of 0.05 or less were considered statistically significant.

\section{RESULTS}

No differences were observed in the basic health characteristics between the two age-matched groups (over 65 years old), except for PSA, as summarized in Table-1. Most of the patients were sedentary 
Table 1 - A comparison of the disease variables between the two groups.

\begin{tabular}{lcccc}
\hline & \multicolumn{2}{c}{ Mean \pm SD } & T & p Value \\
\hline & Group I $(\mathrm{n}=42)$ & Group II $(\mathrm{n}=130)$ & & \\
Age (years) & $71.48 \pm 6.80$ & $70.75 \pm 6.10$ & -0.648 & 0.518 \\
Serum PSA (ng/mL) & $17.12 \pm 14.93$ & $9.65 \pm 9.61$ & -3.042 & 0.004 \\
BMI $\left(\mathrm{kg} / \mathrm{m}^{2}\right)$ & $23.80 \pm 2.94$ & $22.98 \pm 2.60$ & -1.724 & 0.087 \\
s-Testosterone (ng/mL) & $3.14 \pm 1.42$ & $3.30 \pm 1.66$ & -1.822 & 0.061 \\
BMD, T-score (mean $\pm \mathrm{SD})$ & $-2.66 \pm 3.20$ & $-1.96 \pm 3.35$ & 1.193 & 0.235 \\
Smoking (\%) & 22.69 & 21.42 & 3.345 & 0.601 \\
\hline
\end{tabular}

Group I = prostate cancer group; Group II = benign prostate hyperplasia group; PSA = prostate specific antigen; BMI = body mass index; $B M D=$ bone mineral density; Dependent variables $=$ T-score.

and did not engage in physically demanding sports or recreational activity, but only in light exercise, such as short walks. Among the former and current smokers, the pack years ranged from 5 to 62 . Additional information on the prostate cancer disease variables for group I is summarized in Table-2.

\section{The BMD between the Two Groups}

No significant difference was detected in the prevalence of bone loss between the two groups. In group I, $69.05 \%$ had osteopenia (16.67\%) or osteoporosis $(52.38 \%)$ of the spine, while in group II, $55.38 \%$ had osteopenia $(9.23 \%)$ or osteoporosis $(46.15 \%)$. The estimated mean T-score was higher in group I than in group II $(-1.96 \pm 3.35$ vs. $-2.66 \pm 3.20)$, but the difference was not statistically significant $(p=0.235)$

Table 2 - Basic characteristics of group I (prostate cancer group).

\begin{tabular}{lc}
\hline Characteristics & $\mathbf{N}(\%)$ \\
\hline Prostate cancer stage & \\
cT1NxM0 & $18(42.8)$ \\
cT2NxM0 & $22(25.4)$ \\
cT3NxM0 & $2(4.8)$ \\
Gleason's score & \\
$3+3=6$ & $4(9.5)$ \\
$7(4+3,3+4)$ & $16(38.1)$ \\
$4+4=8$ & $12(28.6)$ \\
$9(4+5,5+4)$ & $10(28.9)$ \\
\hline
\end{tabular}

(Table-1). For all of the participants in this study, old age $(\mathrm{r}=-0.371, \mathrm{p}<0.001)$, a high PSA $(\mathrm{r}=-0.209$, $\mathrm{p}=0.006)$, and low BMI $(\mathrm{r}=0.226, \mathrm{p}=0.003)$ were significantly correlated with bone loss.

\section{The BMD in Patients in Group-I}

The mean patient age was 71.48 years. The number (\%) of participants by clinical stage T1, T2, and T3 was 18 (42.8), 22 (25.4), and 2 (4.8) respectively and by a Gleason's score of $6,7,8$, and 9 was 4 (9.5), 16 (38.1), 12 (28.6), and 10 (28.9), respectively. Of those with prostate cancer, $69.05 \%$ had osteopenia $(16.67 \%)$ or osteoporosis $(52.38 \%)$ of the spine (mean T-score $-2.66 \pm 3.20$ ). The significant factors correlated with BMD in group I were a high serum $\operatorname{PSA}(\beta=-0.346, p=0.010)$ and low BMI $(\beta=0.345$, $\mathrm{p}=0.014)$ in the multiple linear regression model (Table-3). Also an old age $(\mathrm{r}=-0.481, \mathrm{p}=0.001)$, a high serum PSA $(r=-0.571, \mathrm{p}<0.001)$, low BMI $(\mathrm{r}$ $=0.598, p<0.001)$, and a high Gleason's score $(\mathrm{r}=$ $-0.319, p=0.040)$ were the factors related with BMD in the univariate analysis (Table-4).

\section{The BMD in Patients in Group-II}

The mean patient age was 70.7 years. Of those with BPH, $55.38 \%$ had osteopenia $(9.23 \%)$ or osteoporosis $(46.15 \%)$ of the spine (mean T-score $-1.96 \pm 3.35)$. The significant factors correlated with BMD in group II were old age $(\beta=-0.324, p=0.001)$ and BMI $(\beta=0.143, p=0.014)$. Smoking, serum testosterone and clinical stage were not significantly correlated with BMD (Table-5). 
Table 3 - The factor associated with bone loss in Group I (prostate cancer).

\begin{tabular}{lccc}
\hline & Standardized Coefficients & T & p Value* \\
\hline & Beta & & \\
Age (years) & -0.228 & -1.797 & 0.081 \\
Smoking $(\%)$ & -0.040 & -0.334 & 0.741 \\
Serum PSA (ng/mL) & -0.346 & -2.729 & 0.010 \\
BMI $\left(\mathrm{kg} / \mathrm{m}^{2}\right)$ & 0.345 & 2.587 & 0.014 \\
Serum Testosterone $(\mathrm{ng} / \mathrm{mL})$ & 0.095 & 0.711 & 0.482 \\
Clinical stage & 0.032 & 0.269 & 0.790 \\
Gleason's score & -0.120 & -0.991 & 0.328 \\
\hline
\end{tabular}

Adjusted $R^{2}=0.479 ;$ Dependent variables $=T$-score; $*$ calculated from multiple linear regression model.

Table 4 - The factors associated with bone loss in Group I (prostate cancer group).

\begin{tabular}{lccccccc}
\hline & T-score & $\begin{array}{c}\text { Age } \\
\text { (years) }\end{array}$ & $\begin{array}{c}\text { Smoking } \\
(\%)\end{array}$ & $\begin{array}{c}\text { s-PSA } \\
(\mathrm{ng} / \mathrm{mL})\end{array}$ & $\begin{array}{c}\text { BMI } \\
\left(\mathrm{kg} / \mathrm{m}^{2}\right)\end{array}$ & $\begin{array}{c}\text { s-Testosterone } \\
(\mathrm{ng} / \mathrm{mL})\end{array}$ & $\begin{array}{c}\text { Clinical } \\
\text { Stage }\end{array}$ \\
\hline Age (years) & $-0.481^{* *}$ & & & & & & \\
Smoking $(\%)$ & 0.033 & -0.155 & & & & & \\
s-PSA $(\mathrm{ng} / \mathrm{mL})$ & $-0.571^{* *}$ & 0.296 & -0.143 & & & & \\
BMI $\left(\mathrm{kg} / \mathrm{m}^{2}\right)$ & $0.598^{* *}$ & $-0.355^{*}$ & -0.081 & $-0.391^{*}$ & & & \\
s-Testosterone $(\mathrm{ng} / \mathrm{mL})$ & 0.114 & -0.086 & -0.215 & 0.035 & 0.027 & & \\
Clinical stage & 0.157 & -0.154 & -0.175 & -0.034 & 0.238 & 0.080 & \\
Gleason's score & $-0.319^{*}$ & 0.244 & -0.171 & 0.223 & -0.221 & $-0.364^{*}$ & 0.092 \\
\hline
\end{tabular}

$*{ }^{*} p<0.01 ; * p<0.05$; calculated from Spearman's correlation analysis; Dependent variables $=T$-score.

Table 5 - The factors associated with bone loss in Group II (benign prostatic hyperplasia group.

\begin{tabular}{lccc}
\hline & $\begin{array}{c}\text { Standardized Coefficients } \\
\text { Beta }\end{array}$ & T & p Value* \\
\hline Age (years) & -0.324 & -4.216 & 0.001 \\
Serum PSA $(\mathrm{ng} / \mathrm{mL})$ & -0.035 & -0.475 & 0.635 \\
BMI $\left(\mathrm{kg} / \mathrm{m}^{2}\right)$ & 0.143 & 1.794 & 0.014 \\
Smoking & 0.113 & 1.496 & 0.075 \\
Serum Testosterone $(\mathrm{ng} / \mathrm{mL})$ & 0.021 & 0.274 & 0.784 \\
\hline
\end{tabular}

Adjusted $R^{2}=0.118$; dependent variables $=T$-score; $*$ calculated from multiple linear regression model.

\section{COMMENTS}

As the prevalence of prostate cancer and osteoporosis increases with age, many patients may already have osteoporosis when diagnosed with prostate cancer. Orchiectomy and the administration of a gonadotropin-releasing hormone agonist, which is the main treatment for metastatic prostate cancer, 
have been reported to cause significant bone loss and lead to bone fracture (7). This is of great concern for men with prostate cancer who will receive ADT (8). Therefore, osteoporosis should be prevented in men with prostate cancer who may require ADT.

Prostate cancer produces and secretes abundant PSA, which is not synthesized in other tumors or tissues. PSA is an important, widely used serologic marker for prostate cancer, but its role in bone metastases is still unclear. PSA, a serine protease, and matrix metalloproteinases are involved in the breakdown of the extracellular matrix that promotes the invasion and metastasis of tumor cells in bone (9). In addition, elevated serum PSA levels are associated with advanced prostate cancer, and prostate cancer cells stimulate the release of various cytokines, which activate osteoclasts and bone resorption (10). Prostate cancer preferably metastasizes to bone and produces primarily osteoblastic phenotypes, unlike other cancers, which are associated with osteoclast formation. Among the known osteogenic factors produced by prostate cells, bone morphogenic proteins, endothelin-1, insulin-like growth factors, parathyroid hormone-related peptide, transforming growth factor$\beta$, and PSA, the latter is uniquely produced by prostate cancer cells (11-16). Men with prostate cancer with poorly differentiated cells and a high Gleason's score have lower testosterone levels than those with well differentiated cells and a low Gleason's score (17). Generally, poorly differentiated prostate cancer is very progressive and metastasizes rapidly; the clinical stage is already high at diagnosis.

Although aging and a low BMI are known risk factors for osteoporosis, whether the serum PSA level or Gleason's score are risk factors for osteoporosis remains unclear in men with prostate cancer. In this study sample, a low BMI and elevated serum PSA levels were significant factors of decreased BMD in the multivariate analysis. Also, old age, a low BMI, elevated serum PSA levels, and a high Gleason's score were significantly associated with bone loss in men with prostate cancer, in the univariate analysis. These results suggest that men with prostate cancer, who are slender and have higher serum PSA levels, are at increased risk of developing a decreased BMD after ADT. In this study, the total serum testosterone was not different between the two groups and was not significantly correlated with BMD. In addition, no correlation with bone loss was observed with the clinical stage of the disease. Although smoking causes osteoporosis, no correlation with bone loss was detected in our study sample, differing somewhat from our previous prediction. This may have been caused by the relatively small number of patients in this study sample.

To date, no convincing study on the status of BMD in non-metastatic prostate cancer prior to ADT had been conducted in Korea. In the present study, $69.05 \%$ of the patients with non-metastatic prostate cancer had osteopenia (16.67\%) or osteoporosis (52.38\%) of the spine before ADT, which is similar to another study in which $73.5 \%$ had osteopenia (55.9\%) or osteoporosis (17.6\%) of the spine (8).

One of the limitations of this study is that the exact intake of calcium and vitamin D, as well as smoking status and the type of daily activities, which are other factors potentially affecting BMD, were not considered. For accuracy, a future study must include all of these factors. In addition, the small size of the non-metastatic prostate cancer group in this study is a limitation. Many studies have recommended that one should check the baseline BMD in all men before starting ADT when osteoporotic risk factors are found $(18,19)$. One should also consider performing BMD studies in older men who have a high serum PSA and a slender stature before initiating ADT in prostate cancer.

\section{CONCLUSIONS}

The risk factors for osteoporosis in men with prostate cancer include old age, a low BMI, and elevated serum PSA. Consideration should be given to performing BMD studies in these men before initiating $\mathrm{ADT}$ in prostate cancer. Monitoring BMD from the outset of ADT is a logical first step in the clinical strategy to avoid or minimize potential bone-related complications in these patients.

\section{CONFLICT OF INTEREST}

None declared. 


\section{REFERENCES}

1. Orwoll E, Ettinger M, Weiss S, Miller P, Kendler D, Graham J, et al.: Alendronate for the treatment of osteoporosis in men. N Engl J Med. 2000; 343: 60410.

2. Daniell HW: Osteoporosis after orchiectomy for prostate cancer. J Urol. 1997; 157: 439-44.

3. Hatano T, Oishi Y, Furuta A, Iwamuro S, Tashiro K: Incidence of bone fracture in patients receiving luteinizing hormone-releasing hormone agonists for prostate cancer. BJU Int. 2000; 86: 449-52.

4. Oefelein MG, Ricchuiti V, Conrad W, Seftel A, Bodner D, Goldman H, et al.: Skeletal fracture associated with androgen suppression induced osteoporosis: the clinical incidence and risk factors for patients with prostate cancer. J Urol. 2001; 166: 1724-8.

5. Mittan D, Lee S, Miller E, Perez RC, Basler JW, Bruder JM: Bone loss following hypogonadism in men with prostate cancer treated with GnRH analogs. J Clin Endocrinol Metab. 2002; 87: 3656-61.

6. Blake GM, Fogelman I: Principles of bone densitometry. In: Bilezikian JP, Raisz LG, and Rodan GA (ed.), Principles of Bone Biology. San Diego, Academic Press. 1996; pp. 1313-32.

7. McGrath SA, Diamond T: Osteoporosis as a complication of orchiectomy in 2 elderly men with prostatic cancer. J Urol. 1995; 154: 535-6.

8. Conde FA, Sarna L, Oka RK, Vredevoe DL, Rettig $\mathrm{MB}$, Aronson WJ: Age, body mass index, and serum prostate-specific antigen correlate with bone loss in men with prostate cancer not receiving androgen deprivation therapy. Urology. 2004; 64: 335-40.

9. Stetler-Stevenson WG, Aznavoorian S, Liotta LA: Tumor cell interactions with the extracellular matrix during invasion and metastasis. Annu Rev Cell Biol. 1993; 9: 541-73.

10. Ershler WB, Harman SM, Keller ET: Immunologic aspects of osteoporosis. Dev Comp Immunol. 1997; 21: 487-99.

11. Komori T, Yagi H, Nomura S, Yamaguchi A, Sasaki $\mathrm{K}$, Deguchi K, et al.: Targeted disruption of Cbfal results in a complete lack of bone formation owing to maturational arrest of osteoblasts. Cell. 1997; 89: 755-64.

12. Yang J, Fizazi K, Peleg S, Sikes CR, Raymond AK, Jamal N, et al.: Prostate cancer cells induce osteoblast differentiation through a Cbfal-dependent pathway. Cancer Res. 2001; 61: 5652-9.

13. Komori T: Runx2, a multifunctional transcription factor in skeletal development. J Cell Biochem. 2002; 87: $1-8$.

14. Karsenty G: The genetic transformation of bone biology. Genes Dev. 1999; 13: 3037-51.

15. Yingling JM, Blanchard KL, Sawyer JS: Development of TGF-beta signalling inhibitors for cancer therapy. Nat Rev Drug Discov. 2004; 3: 1011-22.

16. Bonewald LF, Mundy GR: Role of transforming growth factor-beta in bone remodeling. Clin Orthop Relat Res. 1990; 250: 261-76.

17. Eriksson A, Carlström K: Prognostic value of serum hormone concentrations in prostatic cancer. Prostate. 1988; 13: 249-56.

18. Higano CS: Management of bone loss in men with prostate cancer. J Urol. 2003; 170: S59-63; discussion S64.

19. Conde FA, Aronson WJ: Risk factors for male osteoporosis. Urol Oncol. 2003; 21: 380-3.

Accepted after revision: December 3, 2008

\section{Correspondence address:}

Dr. Taek Won Kang

Department of Urology

Chonnam National University Med. Sch.

8, Hak-dong, Dong-gu

Gwangju \#501-757, Republic of Korea

Fax: + 8262 227-1643

E-mail: sydad@hanmail.net 


\section{EDITORIAL COMMENT}

This is an interesting paper describing risk factors for osteopenia in men with prostate cancer and benign prostatic hyperplasia undergoing androgen deprivation therapy (ADT). The work describes high prostate specific antigen and low body mass index as risk factors for men with prostate cancer about to undergo ADT. It is important to screen such men prior to ADT to determine if further steps are needed, such as vitamin D and calcium supplementation or bisphosphonate treatment.

Dr. A. M. Brufsky Magee-Womens Hospital University of Pittsburgh School of Medicine Pittsburgh, Pennsylvania, USA E-mail:brufskyam@upmc.edu 